\title{
On the Trade-off between Electrical Power Consumption and Flight Performance in Fixed-Wing UAV Autopilots
}

\author{
Eduard Bertran, Senior Member, IEEE, and Alex Sànchez-Cerdà
}

\begin{abstract}
This paper sets out a study of the autopilot design for fixed wing UAVs taking into account the aircraft stability, and also the power consumption as a function of the selected control strategy. To provide some generality to the outcomes of this study, construction of a reference small-UAV model, based on averaging the main aircraft defining parameters, is proposed. Using such a reference model of small, fixed-wing UAVs, different control strategies are assessed especially with a view towards enlarging the controllers' sampling time. A beneficial consequence of this sample time enlargement is that the clock rate of the UAV autopilots may be proportionally reduced. This reduction in turn leads directly to decreased electrical power consumption. Such energy saving becomes proportionally relevant as the size and power of the UAV decreases, with benefits of lengthening battery life and, therefore, the flight endurance. Additionally, through the averaged model, derived from both published data and computations made from actual data captured from real UAVs, it is shown that behavior predictions beyond that of any particular UAV model may be extrapolated.
\end{abstract}

Index Terms - Unmanned aerial vehicles, aircraft systems, control systems, autopilots, CMOS electronics, power consumption.

\section{INTRODUCTION}

The growing interest in Unmanned Aerial Vehicles (UAVs) has been steered by political, economic, security and other such drivers. Compared to conventionally piloted aircraft, smallmedium size UAVs are significantly cheaper, easier to exploit, benefit more readily from technological developments, and show better maneuverability in some complicated or unsafe scenarios. For some specific telecommunication systems applications, such as ad hoc special purpose applications, UAVs with large wing spans [1] or even those based on balloons [2] offer some advantages over conventional solutions such as GEO, MEO or LEO satellites. Moving beyond initial military and security applications (e.g., border reconnaissance, surveillance operations, natural disaster support infrastructure), UAVs are playing a valuable, growing and pervasive role in an ever widening, evolving and diverse range of data collection applications in many spheres of civil life.

While there is no agreed or unique classification of UAVs,

Manuscript received ........

E. Bertran and A. Sanchez are with the Department of Signal Theory and Communications, Technical University of Catalonia. C. Esteve Terrades, 7, 08860 Castelldefels, Barcelona, Spain. (e-mail: bertran@tsc.upc.edu,; alexandre.sanchez.cerda@estudiant.upc.edu). This work was partially supported by the Government of Catalonia, under grant 014 SGR 1103. Digital Object Identifier .... operational range can be classifying attribute. NATO's Joint Capability Group on UAV has made a classification ranging from micro UAVs (below $5 \mathrm{~km}$ ) to High Altitude Long Endurance (HALE) UAVs, which have more than one day of autonomy and operate at altitudes above $14 \mathrm{~km}$. In this paper, we will use the term 'small UAVs' in a wide sense, including UAVs with ranges of up to $50 \mathrm{~km}$.

UAV nomenclature often relates to the kind of operation. Unmanned Aircraft Systems (UAS) is an International Civil Aviation Organization (ICAO) term that refers to a wider concept than just UAVs, and includes remote control supported by ground stations. Unmanned free balloons are also classified as UAS in ICAO documents. Another name is Remotely Piloted Aircraft Systems (RPAS). UAVs of another type are small aircraft which can be rapidly deployed without the need for an airstrip, thus providing early rapid response in search and rescue missions.

Different technological factors have contributed to boosting the usage of unmanned aircraft. The most prominent are the developments of high density batteries, long range radio devices with improved power efficiency, cheaper airframes, and powerful motors, as well as ever improving 'cost-sizeperformance' figures of merit of the microelectronics that constitute the core of the avionics subsystem.

Navigation which refers to the aircraft location and trajectory may be more or less complicated according to the flying scenario. Factors include segregated or non-segregated airspace, cooperative UAV formation [3] or not, constant or changing path, etc. At a lower level, the role of the autopilot is to apply suitable control actions dynamically to assure a correct navigation along the desired waypoints. The autopilot entities are the major and core component of the UAV avionics. They are composed of a host of computing and sensing subsystems. These include the onboard computer; micro inertial guidance utilities comprising gyros, acceleration and magnetic sensors; a GPS receiver; battery monitoring and power distribution board; and an onboard camera [4]. Functionally, the autopilot is the closed-loop control system in charge of the longitudinal and lateral controls for controlling the altitude, forward and vertical speeds, the pitch attitude, and for maintaining aircraft direction and stability within a bank angle. The autopilot algorithm continuously generates commands to the main control surfaces (ailerons, elevator and rudder) and to the throttle to control the motor speed. Small UAVs may not have all these control possibilities.

Autopilots have different operating modes, depending on the degree of autonomy to control the flight surfaces. The set-points to the control surfaces servos may be autonomously computed 
(on board) or relayed through a radio-control link from a Ground Control Station (GCS) [5,6]. The normal operation is to operate autonomously when the objective is just to move the flight surfaces to control the aircraft dynamics between waypoints, these being obtained through a radio link. However, when the waypoints are spaced or when the UAV is faced with unexpected situations, then the autopilot may be required to autonomously compute extra waypoints. It does this in the former situation to reduce power consumption in the onboard radio equipment, as well as to release bandwidth in the radio channel for payload applications; and in the latter, to create alternative routes to handle or avoid unexpected disturbances or encounters in the nominal path. It is these kinds of autonomous autopilot control mode $[4,5,7]$ that are considered in this paper.

The UAV power consumption depends on a variety of factors but mainly on wing loadings, weight and desired performance. A small electrical UAV typically needs around 20 to 200 watts $/ \mathrm{kg}$ to fly. For example, an electrical UAV with $5 \mathrm{~m}$ of wingspan can need a power of 100 watts for a Take-Off Weight (TOW) of $4.5 \mathrm{~kg}$. A value of 200 to 300 watts $/ \mathrm{kg}$ is suited to heavier or higher performance UAVs, growing to $1.5 \mathrm{~kW}$ for $300 \mathrm{~kg}$ TOW. HALE UAVs with their large wing spans of 15 to $50 \mathrm{~m}$ require some tens of $\mathrm{kW}$ of power.

In [8], some UAV power requirements are presented. For a lightweight UAV, the power requirement for up to 2 hours of endurance is typically 40 watts. This power demand can be met by using photovoltaic cells or lithium polymer batteries.

Motors are the main power consuming devices among the entire set of UAV subsystems. The other power hungry subsystems are the power amplifier in the radio transmitter (only for enlarged communication ranges) and the autopilot. The power consumption of the autopilot, typically ranging from 1.5 to 5 watts, while negligible in large aircraft, becomes as relevant as the UAV size shrinks [4]. For instance, in reduced endurance small UAVs the autopilot accounts for up to $5 \%$ of the whole aircraft power, growing to $10 \%$ in lightweight UAVs. Hence, the growing interest in finding battery power consumption reduction measures.

Integrated autopilots for small UAVs are readily available in the market at reasonable prices. Usually they include RF communications, navigation sensors and GPS connections. The autopilot hardware is based on devices usually made in CMOS technology, such as Digital Signal Processors (DSP) or Digital Signal Controllers (DSC) running at clock frequencies of tens of $\mathrm{MHz}[5,8]$ and so executing tens of millions of instructions per second (MIPS). The reduction of the clock frequency shrinks the autopilot power consumption, because of the strong power consumption dependence of CMOS devices to the switching frequency of the internal transistors. Typically the data taken from telemetry and from the internal GPS receiver is updated at a frequency of $5 \mathrm{~Hz}$, while the processing frequency of the control algorithms (sampling frequency of the digital controllers) ranges from 20 to $200 \mathrm{~Hz}[4,5,9,10,11]$.

The elementary autopilot control facilitates a manual override by a remote pilot. While classical PID is the most common autopilot control strategy encountered for automatic control of the flight surfaces, more advanced strategies, such as neural networks, fuzzy logic, adaptive structures, $\mathrm{LQR}$ or $\mathrm{H}_{-\infty}$ have been proposed. There are a variety of trade-offs among these strategies. For instance, some have reduced accuracy requirements of the dynamical model of the aircraft in order to compute good controller parameters, while others have better responses (stability and/or precision) in face of external disturbances but this at the cost of detailed accurate aircraft design models. Additional trade-off elements are the control algorithm sampling time and the necessity of internal control loops. The sampling time directly affects the sampling frequency in the DSP device. As indicated, device power consumption is proportional to the clock rate. Also, the use of additional inner control loops requires the use of more variables (more sensors), with the usual redundancy.

In the study presented here different autopilot control strategies are evaluated with respect to their sensitivity to increasing the sampling period. To our knowledge there is no literature addressing this sensitivity, and consequently, the quantitative advantages or disadvantages of a given control algorithm in terms of power consumption. The control strategies considered here are PID, LQG/LQR -the strategy most employed in recent research papers-, and an advanced strategy based on a direct adaptive controller following a model reference structure (MRAS). Results are assessed by considering both the flight quality and the practical implementation needs.

Normally, in published research work showing the benefits of a selected control law, results are underpinned by simulations carried out using a particular UAV model. Given the variety of UAVs, this particularity of the simulation results presents difficulties when it comes to making a comparative analysis of the relative advantages and disadvantages among the different control laws. As a way of dealing with this, we employ what we refer to as an 'averaged model' of reduced size fixed-wing UAVs. This model is extracted from actual UAVs with wingspans lower than $6 \mathrm{~m}$, but not including micro UAVs. To elaborate this 'averaged model' we use the UAV models and their parameters directly obtained from the published technical papers, data sheets and such like documentation sources. For those UAVs for which published dynamic models are unavailable, we have used the USAF Digital Datcom (DATCOM) software [12,13] to compute the parameters of actual UAVs, i.e., from actual UAVs structural data. For the sake of simplicity, this work is based on longitudinal models. This approach is sufficient for our purpose of assessing the control law algorithms' dependency on the sampling period. Depending on the control algorithm under consideration, the models are developed in the state-space or as a simple SISO transfer function.

In the presented study it will be shown the little robustness of the LQR in front of sampling period enlargements, as well as the capability of both the PID and the proposed MRAS regulator to reduce power consumption between 5 and 10 times, keeping a right dynamics in precision and an acceptable setting time (lower than 10s in the adaptive strategy).

\section{MODELS OF FIXED-WING UAVS}

The primary step for designing and assessing control strategies is to have a suitable model. However, for a general study of such strategies, there is a risk in using a model aligned too closely to a particular UAV, in that results and conclusions will in fact lack generality. Even just considering fixed and 
rigid wing UAVs, there can be quite a variation, e.g., relevant differences in the airframe: wingspan, chord, simple or double rudder, V-tail or not, tailboom assemblies, location of motors, fuel or electric propulsion, as well as differences in the materials: metallic, plastic, carbon fiber, etc. Hence our proposition above of an 'averaged model' which we made using both data from two sources as indicated above. We elaborate on this in the follow two subsections.

\section{A. Models from Research Papers}

In the literature proposing autopilot control strategies, different models of UAVs may be found. These are usually presented in state-state format. The first drawback encountered, in seeking to make an averaged model from this data, is the diversity of state vectors (variables) considered in the different papers. This drawback would be easily be overcome if the objective were to elaborate a classical transfer function (SISO model) because the transformation from the state-state representation to the transfer function is quite trivial. However, if the objective is to obtain a generalized state-state model, then solutions to this problem are not easy, are not direct and, besides, most of the techniques to modify or reduce models (e.g., SVD) only preserve some characteristics of the original system (e.g., stability). In our study, we wish to assess and compare, for the same aircraft, some control strategies that only need the transfer function model and other strategies that require a model in the space-state (LQG/LQR). Hence it is necessary to limit the use of published models to those sharing the same state variables. A consequence of such restrictions is the reduction of the number of UAVs to be used in the averaged model. The aircraft considered and selected have wingspans ranging from 2.4 to 5.8 meters and show only slight variations in their geometry. For one of them [14], the model has been made with three different techniques (analytical, grey-box method and black box method), with remarkable differences in its parameters among these techniques. For another two models $[15,16]$, only three of the four considered variables agree. These four variables are longitudinal velocity, $\mathrm{u}$; vertical velocity, w; pitch rate $\rho$; and the pitch angle, $\theta$.

\section{B. DATCOM based models}

The longitudinal aircraft model from the classic book of J. H. Blakelock [17] is adequate for use with the USAF Digital Datcom (DATCOM) software [12,13]. This model is based on the aircraft rigid body equations, using the three degree-offreedom longitudinal equations which, along with the stability derivatives, provide the transfer functions of the aircraft for an elevator input [17]. In the used aircraft axis system the center of coordinates is located at the center of gravity of the aircraft (they may be related with the earth axis -inertial frame- by means of the Euler angles).

As usual, we make the following assumptions: i) the mass of the aircraft remains constant, ii) the aircraft is a rigid body, iii) the earth is an inertial reference, iv) the perturbations from equilibrium are small, and v) the flow is quasi-steady.

The model considers three variables: the speed in the longitudinal direction $(\mathrm{u})$, the angle of attack $(\alpha)$ and the pitch angle $(\theta)$. By applying dynamic principles to the plane, with the usual assumptions of constant mass and atmosphere and aircraft symmetries, after balancing steady-state aerodynamic forces and moments in the aircraft body, expanded in terms of the changes in them and supposing small displacements (smallangle approximations on the transformation from the earth axis to the aircraft axis), the classical [17] longitudinal equations of motion can be obtained in the s-domain (Laplace transform):

$$
\begin{aligned}
& \left(\frac{m U}{S q} \cdot s-C x u\right) \cdot u(s)-C x \alpha \cdot \alpha(s)-C w \cdot \theta(s)=0 \\
& -C z u \cdot u(s)+\left(\left(\frac{m U}{S q}-\frac{c}{2 U} \cdot C z \dot{\alpha}\right) \cdot s-C z \alpha\right) \cdot \alpha(s)+ \\
& +\left(\left(-\frac{m U}{S q}-\frac{c}{2 U} \cdot C z q\right) \cdot s\right) \cdot \theta(s)=C z \delta e \cdot \delta e(s) \\
& \left(-\frac{c}{2 U} \cdot C m \dot{\alpha} \cdot s-C m \alpha\right) \cdot \alpha(s)+ \\
& +\left(\frac{I y}{S q c} \cdot s^{2}-\frac{c}{2 U} \cdot C m q \cdot s\right) \cdot \theta(s)=C m \delta e \cdot \delta e(s)
\end{aligned}
$$

\begin{tabular}{|c|c|c|c|}
\hline $\mathrm{u}$ & longitudinal velocity & $\mathrm{q}$ & dynamic pressure \\
\hline $\mathrm{U}$ & $\begin{array}{l}\text { absolute velocity in the } \\
\text { longitudinal direction }\end{array}$ & $\mathrm{c}$ & $\begin{array}{l}\text { mean aerodynamic chord } \\
\text { (MAC) of the wing, }\end{array}$ \\
\hline $\mathrm{w}$ & vertical velocity & $\mathrm{I}_{\mathrm{Y}}$ & $\begin{array}{l}\text { body inertia around y axis } \\
\text { (lateral axis) }\end{array}$ \\
\hline$\alpha$ & angle of attack & $\delta \mathrm{e}$ & elevator displacement \\
\hline$\theta$ & pitch angle & $\begin{array}{l}\mathrm{Cx}, \\
\mathrm{Cw}, \\
\mathrm{Cz} \\
\mathrm{Cm}\end{array}$ & $\begin{array}{l}\text { control and stability } \\
\text { derivatives. } \boldsymbol{C} \boldsymbol{x}_{\boldsymbol{u}} \text { (variation } \\
\text { of thrust with } \mathrm{u}), \boldsymbol{C}_{\boldsymbol{w}} \\
\text { (gravity), } \boldsymbol{C} \boldsymbol{z}_{\boldsymbol{u}} \text { (variation of } \\
\text { normal force with } \mathrm{u} \text { ) }\end{array}$ \\
\hline$\rho=\dot{\theta}$ & pitch rate & $\mathrm{m}$ & mass of the aircraft \\
\hline $\mathrm{S}$ & wing surface & $\mathrm{h}$ & altitude \\
\hline
\end{tabular}

The aerodynamic parameters used along this paper are tabulated in Table I

TABLE I

TABULATED AERODYNAMIC PARAMETERS

To represent the entry of the system (excitation), the elevator displacement $\delta$ e has been added. The derivatives $\mathrm{Cx}, \mathrm{Cw}, \mathrm{Cz}$ and $\mathrm{Cm}$ may be computed by using the DATCOM software.

In order to simplify the application of these equations to design some control techniques, they are converted to statespace form $[17,18]$. To do it, the pitch rate $\dot{\theta}$ has to be included as additional variable, $\rho=\dot{\theta}$.

$$
\left[\begin{array}{c}
\dot{u} \\
\dot{\alpha} \\
\dot{\theta} \\
\dot{\rho}
\end{array}\right]=A \cdot\left[\begin{array}{l}
u \\
\alpha \\
\theta \\
\rho
\end{array}\right]+B \cdot \delta e
$$

where $\mathrm{B}$ is a $4 \mathrm{x} 1$ matrix and $\mathrm{A}$ is a $4 \mathrm{x} 4$ matrix.

$$
B=\left[\begin{array}{c}
0 \\
\frac{C z \delta e}{\frac{m U}{S q}-\frac{c}{2 U} C z \dot{\alpha}} \\
0 \\
\frac{c}{2 U} C m \dot{\alpha} \\
\frac{C z \delta e}{\left(\frac{I y}{S q c}\right)} \cdot \frac{C m U}{\frac{m U}{S q}-\frac{c}{2 U} C z \dot{\alpha}}+\frac{C m e}{\left(\frac{I y}{S q c}\right)}
\end{array}\right]
$$




$$
A=\left[\begin{array}{cccc}
\frac{C x u}{\frac{m U}{S q}} & \frac{C x \alpha}{\frac{m U}{S q}} & \frac{C w}{m U} & 0 \\
\frac{C z u}{\frac{m U}{S q}-\frac{c}{2 U} C z \dot{\alpha}} & \frac{C z \alpha}{\frac{m U}{S q}-\frac{c}{2 U} C z \dot{\alpha}} & 0 & \frac{m U}{S q}+\frac{c}{2 U} C z q \\
\frac{c}{2 U} C m \dot{\alpha} \\
\frac{0}{\left(\frac{I y}{S q c}\right)} \frac{C z u}{\frac{m U}{S q}-\frac{c}{2 U} C z \dot{\alpha}} & \frac{c}{\frac{c}{2 U} C m \dot{\alpha}}-\frac{c}{2 U} C z \dot{\alpha} \\
\frac{I y}{S q c} & \frac{C z \alpha}{\frac{m U}{S q}-\frac{c}{2 U} C z \dot{\alpha}}+\frac{C m \alpha}{\frac{I y}{S q c}} & 0 & \frac{\frac{c}{2 U} C m \dot{\alpha}}{\left(\frac{I y}{S q c}\right)} \cdot \frac{\frac{m U}{S q}+\frac{c}{2 U} C z q}{\frac{m U}{S q}-\frac{c}{2 U} C z \dot{\alpha}}+\frac{\frac{c}{2 U} C m q}{\left(\frac{I y}{S q c}\right)}
\end{array}\right]
$$

Four UAVs have been modeled by means of DATCOM, cf. Fig. 1. They are: ALO [19], Siva [19], Megastar, [20,21] and Shadow MK-1 [21]. ALO (span: $3.84 \mathrm{~m}$ and maximum takeoff weight, MTOW: $55 \mathrm{~kg}$ ) and SIVA (span: $5.81 \mathrm{~m}$, maximum weight: $300 \mathrm{~kg}$ ) are UAVs developed by the Spanish National Institute for Aerospace Technology. Megastar 1.50 ARF, manufactured by Proteus, has a wingspan of $2.4 \mathrm{~m}$ and a weight of $5.9 \mathrm{~kg}$ (no MTOW data provided), while Shadow MK-1 is an experimental prototype developed by the ICARUS team [22], with 5.2 meters of wingspan, and allowing a MTOW of $90 \mathrm{~kg}$ (dry weigh of $55 \mathrm{~kg}$ ).

\section{ACTUAL UAVs}
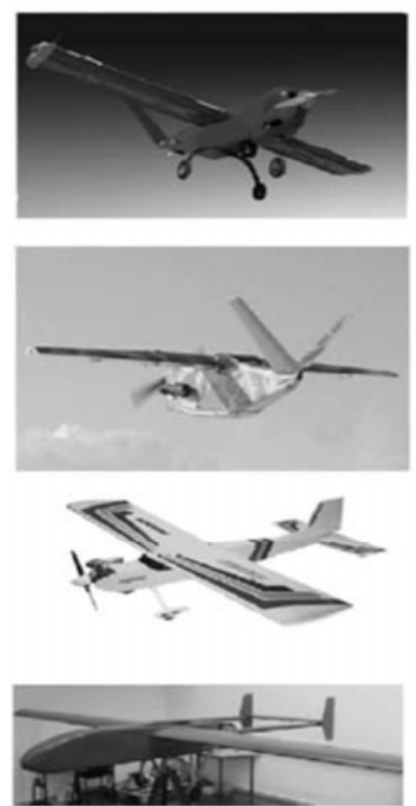

d)

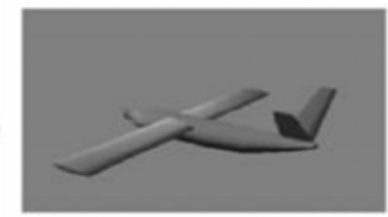

b)

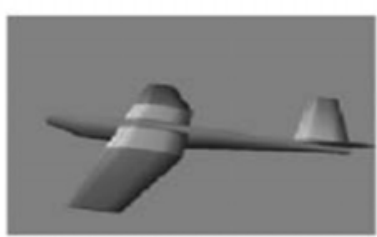

c)
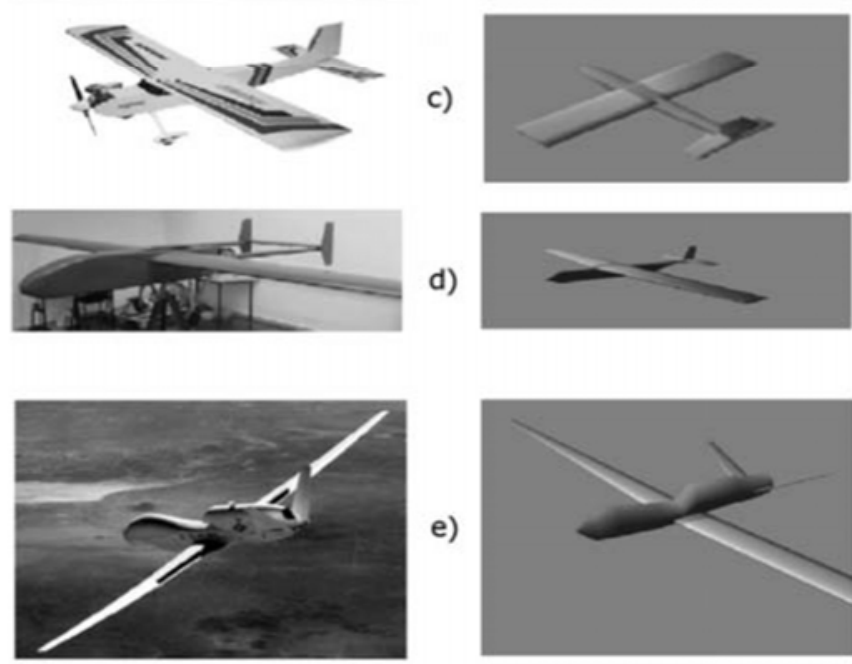

e)

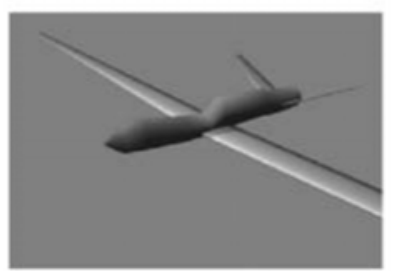

Fig. 1 Some considered UAVs and their DATCOM models: ALO (a), Siva (b), Megastar (c), Shadow (d) and Global Hawk (e).
Initially, two large fixed-winged UAVs were considered for incorporation into the 'averaged model'. These were the Global Hawk [23], with a wingspan of $35.3 \mathrm{~m}$ and the scalesized technological demonstrator (24 m wingspan) of the Heliplat prototype [24]. However, the differences among the parameters of both models compared to those of the lower wingspan models were too significant, and too unbalancing for the generality of the averaged model; we decided to exclude them.

\section{AVERAGED MODEL}

Nine different fixed-wings UAVs models have been used, five from research publications and four elaborated with DATCOM. Because there are differences in the variables used for defining the models, cf. Table II, it is not possible to compute an average of the state-space models by a simply parameter averaging process.

TABLE II

FIXED-WING AIRCRAFT MODELS WITH THEIR CORRESPONDING VARIABLES (DESCRIBED AS INDICATED ABOVE)

\begin{tabular}{|l|c|}
\hline \multicolumn{1}{|c|}{ UAV model } & $\begin{array}{c}\text { Variables of the } \\
\text { model }\end{array}$ \\
\hline BPPT Wulung UAV (analytical) [14] & $\{\mathrm{u}, \mathrm{w}, \rho, \theta\}$ \\
\hline BPPT Wulung UAV (grey-box) [14] & $\{\mathrm{u}, \mathrm{w}, \rho, \theta\}$ \\
\hline BPPT Wulung UAV (black-box) [14] & $\{\mathrm{u}, \mathrm{w}, \rho, \theta\}$ \\
\hline Conventional model 1 [15] & $\{\mathrm{u}, \rho, \theta, \alpha\}$ \\
\hline Conventional model 2 [16] & $\{\mathrm{u}, \rho, \theta, \mathrm{h}\}$ \\
\hline ALO UAV (DATCOM) & $\{\mathrm{u}, \alpha, \theta, \rho\}$ \\
\hline Siva UAV (DATCOM) & $\{\mathrm{u}, \alpha, \theta, \rho\}$ \\
\hline Megastar UAV (DATCOM) & $\{\mathrm{u}, \alpha, \theta, \rho\}$ \\
\hline Shadow UAV (DATCOM) & $\{\mathrm{u}, \alpha, \theta, \rho\}$ \\
\hline
\end{tabular}

Rather, an averaged model may be found by averaging the individual model impulse responses, described by the pole-zero $(p-z)$ locations in the s-domain. The $p-z$ locations of the individual models (real and imaginary terms) are averaged.

Once the locations for the averaged residuals are known, the next step is to solve the inverse problem to obtain the model parameters - control and stability derivatives of equations (1), (2) and (3) - by means of which the poles and zeroes may be placed in the target locations. Special care needs to be taken on the dominant poles (closer to the imaginary axis). These are the slower ones, describing the phugoid motion. The dominant 
poles for the averaged model are located at $s=-0.02474 \pm$ j 0.3191. These poles are also critical in other works which assess the accomplishment of the Reduced Vertical Separation Minimum (RVSM), i.e., assess the ICAO conditions for UAV operation in a non-segregated air-space environment.

After a sensitivity study, we have realized that the poles and zeroes of the transfer function are particularly sensitive to the terms $\frac{\boldsymbol{m} \cdot \boldsymbol{u}}{\boldsymbol{s} \cdot \boldsymbol{q}}$ and $\boldsymbol{C} \boldsymbol{x}_{\boldsymbol{u}}$ (i.e., variation of drag and thrust with $\mathrm{u}$ ). At a secondary level, the transfer function is sensitive to $\boldsymbol{C}_{\boldsymbol{w}}$ (gravity), $\boldsymbol{C} \boldsymbol{z}_{\boldsymbol{u}}$ (variation of normal force with $\mathrm{u}$ ) and $\frac{\boldsymbol{c}}{\boldsymbol{2 \cdot \boldsymbol { U }}}$. The remaining parameters show little sensitivity to modifications to the dominant $\mathrm{p}-\mathrm{z}$ locations. They only affect the residuals related to the fast components of the response. The resulting averaged model equations from DATCOM models are:

$$
\begin{aligned}
& \left(\begin{array}{c}
\dot{u} \\
\dot{\alpha} \\
\dot{\theta} \\
\dot{\rho}
\end{array}\right)=\left[\begin{array}{ccccc}
-0.0353 & 0.0283 & -0.2561 & 0 \\
-0.4003 & -2.4955 & 0 & 0.9819 \\
0 & 0 & 0 & 1 \\
0.2373 & -13.5921 & 0 & -1.9673
\end{array}\right] \cdot\left(\begin{array}{l}
u \\
\alpha \\
\theta \\
\rho
\end{array}\right)+ \\
& +\left(\begin{array}{c}
0 \\
-0.1298 \\
0 \\
-14.2371
\end{array}\right) \cdot \delta e
\end{aligned}
$$

Merging this model with the ones obtained from the published papers yields the final version of the averaged model:

$$
\begin{aligned}
& \left(\begin{array}{c}
\dot{u} \\
\dot{\alpha} \\
\dot{\theta} \\
\dot{\rho}
\end{array}\right)=\left[\begin{array}{cccc}
-0.0688 & 0.0283 & -03098 & 0 \\
-0.4003 & -2.4955 & 0 & 0.9819 \\
0 & 0 & 0 & 1 \\
0.2373 & -13.5921 & 0 & -1.9673
\end{array}\right] \cdot\left(\begin{array}{l}
u \\
\alpha \\
\theta \\
\rho
\end{array}\right)+ \\
& +\left(\begin{array}{c}
0 \\
-0.1298 \\
0 \\
-14.2371
\end{array}\right) \cdot \delta e
\end{aligned}
$$

For the LQR controller, the performance index is defined as usual [18]:

$$
J=\int_{0}^{t f}\left(x^{T} \cdot Q \cdot x+u^{T} \cdot R \cdot u\right) d t
$$

where the weighting matrixes $\mathrm{Q}$ and $\mathrm{R}$ are:

$$
\begin{gathered}
Q=\left[\begin{array}{cccc}
1 / u_{\max } & 0 & 0 & 0 \\
0 & 1 / \alpha_{\max } & 0 & 0 \\
0 & 0 & 1 / \theta_{\max } & 0 \\
0 & 0 & 0 & 1 / \dot{\theta}_{\max }
\end{array}\right] \\
R=\left[1 / \delta_{e_{\max }}\right]
\end{gathered}
$$

The adaptive controller follows the MRAS model [29] shown in the schematics in Fig. 2 and Fig. 3.

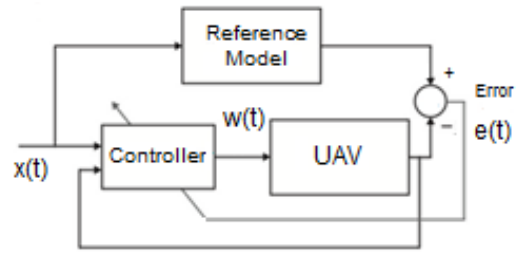

Fig. 2 Direct adaptive control based on a model reference structure.

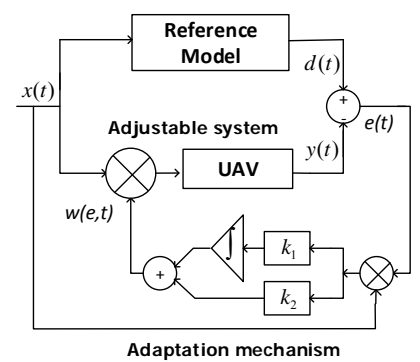

Fig. 3 Direct adaptive control law

And the control law is $[25,26]$ :

$$
w(e, t)=\int_{0}^{t} f_{1}(e, t, \tau) d \tau+f_{2}(e, \tau)
$$

$f_{1}(e, \tau)=k_{1} x(t) e(t) ; f_{2}(\xi, \tau)=k_{2} ; k_{1}, k_{2}>0$

where $f_{1}$ is related to the system precision and $f_{2}$ to the velocity (settling time of the transient response).

The adaptive controller Matlab/Simulink simulation model is shown in Fig. 4.

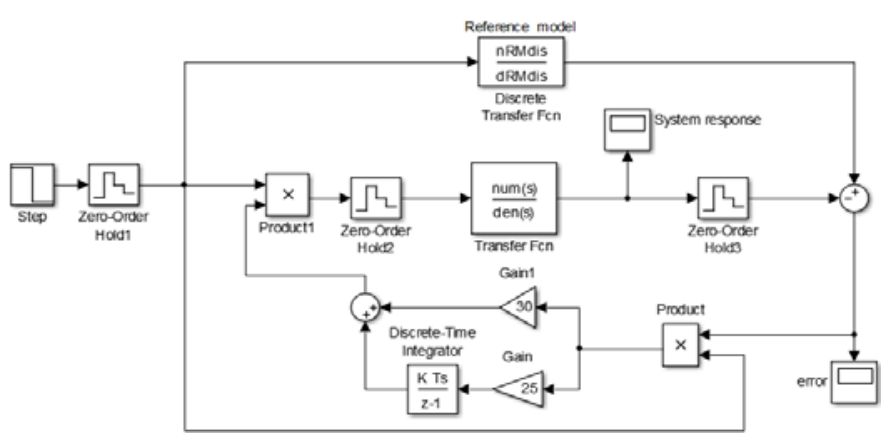

Fig. 4 Adaptive control simulation model for fixed-wing UAVs

In order to also evaluate the robustness of the controllers, the simulations were made for both the obtained averaged model (whose step response can be seen in Fig. 5) and for the same model with a number of parameters under disturbance (Fig.5).

For sampling periods (Ts) up to $1 \mathrm{~s}$, the operational performance of all three controllers was shown to be adequate. Fig. 6 shows the results of the three controllers (pitch angle variation) for $\mathrm{Ts}=1 \mathrm{~s}$. Even with the best empirical adjustment, PID is the slowest to reach the right steady-state angle. However in its favor, and in contrast with LQR which is the quickest, it does not require a detailed model. However, the adaptive controller competes as the best option as it does not need very accurate models and so it represents a good compromise 
between the quality of the response and the required level of model accuracy.

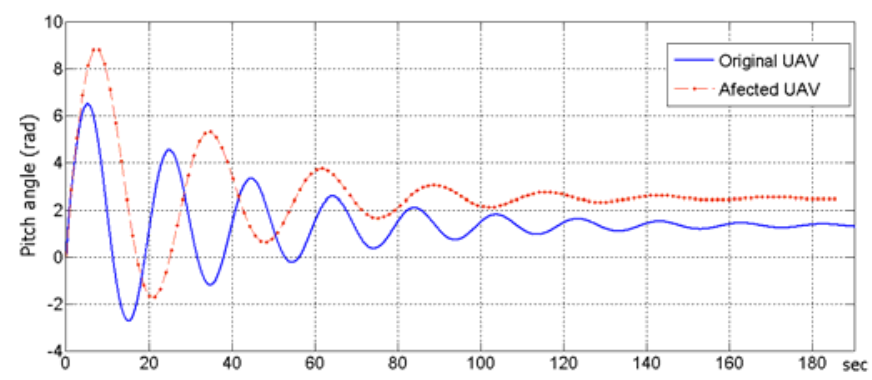

Fig. 5 Step response of the averaged model (solid line) and the disturbed model (dashed line)

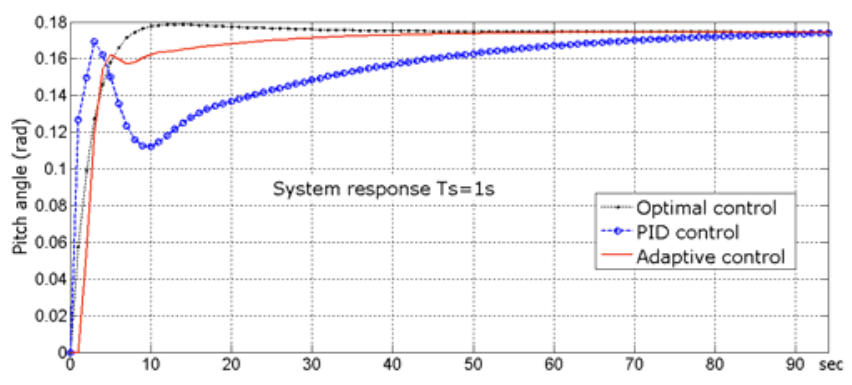

Fig. 6 Comparison of step responses for Ts $=1 \mathrm{~s}$ (pitch angle)

Considering responses to UAV disturbances, as can be seen in Fig 7, LQR fails to compensate for these (leaves the UAV with a significant steady state position error). Conversely, the responses of the PID controller and the MRAS controller, in successfully minimizing the final position error, are good; the former, due especially to its integrative action and, the latter, because of its adaptive behavior.

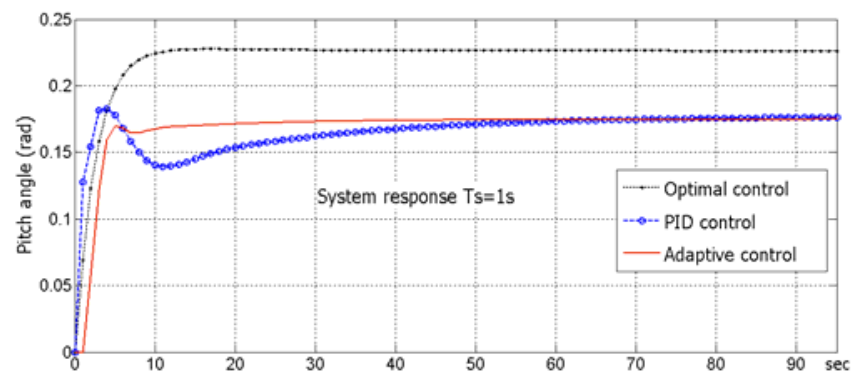

Fig. 7 Step responses of the disturbed aircraft for $\mathrm{Ts}=1 \mathrm{~s}$

As a first attempt to investigate the potential to reduce the battery power consumption, Ts was enlarged to $4 \mathrm{~s}$. The PID fails at this sampling period. Its settling time is increased by up to 10 minutes, which is unacceptable in practice. Both LQR and MRAS still work satisfactorily (Fig. 8). In this study for both controllers the Ts enlargement is not applied as a parametric disturbance. Rather both have been redesigned considering the new sampling period.

Anyway, LQR continues to fail to compensate the dynamics when the UAV is disturbed, while the adaptive controller, it continues to perform well, minimizing the final position error (Fig. 9).

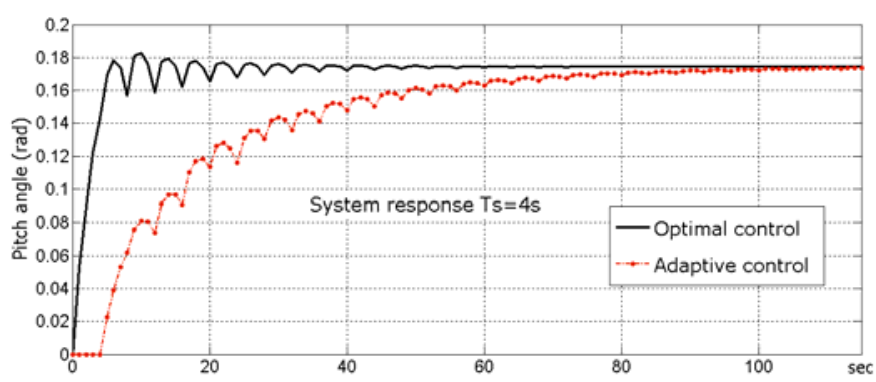

Fig. 8 Optimal and adaptive controllers responses for Ts $=4 \mathrm{~s}$

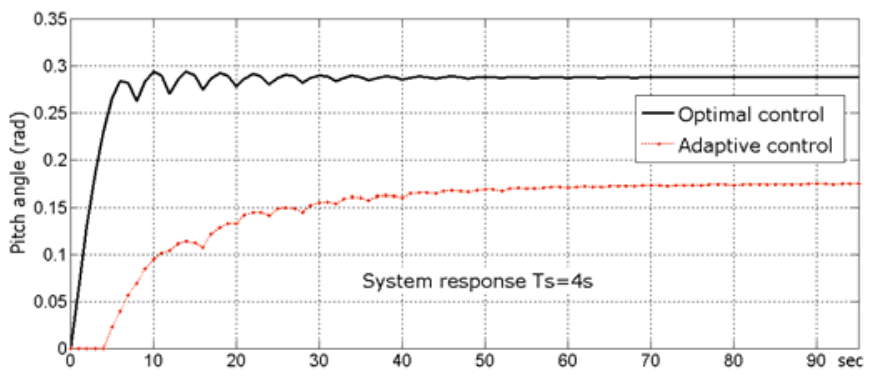

Fig. 9 Disturbed UAV responses for $\mathrm{Ts}=4 \mathrm{~s}$ (adaptive and optimal controllers)

Finally, the parameters of both LQR and the MRAS controllers have been computed for extreme sampling times in order to assess their robustness. MRAS has an acceptable behavior up to Ts $=10 \mathrm{~s}$ (Fig.10), while the LQR accepts redesigns up to $15 \mathrm{~s}$, even though with a low flight quality, i.e., with an excessively underdamped response, Fig. 11.

\section{CMOS POWER CONSUMPTION}

Current autopilot technology is based on CMOS technology [9]. The power consumption of a CMOS device can be estimated [27]:

$$
P_{C}=C_{p d} \cdot V_{c c}^{2} \cdot f_{i} \cdot N_{s w}
$$

where $\mathrm{P}_{\mathrm{C}}$ is the power consumption, $\mathrm{C}_{\mathrm{pd}}$ the dynamic powerdissipation capacitance, $\mathrm{V}_{\mathrm{CC}}$ the supply voltage, $\mathrm{f}_{\mathrm{i}}$ the switching frequency and $\mathrm{N}_{\mathrm{sw}}$ the number of switching bits.

Among other factors, the switching frequency depends on the sampling time required by the UAV's digital control strategy. Hence, and as indicated earlier, by enlarging this time the processor workload and the power consumption will decrease and, as per (13), will do so linearly with the sampling period enlargement.

In the simulation studies shown in previous section, the capability of the different control strategies to support large sampling periods has been assessed. The PID controller has some advantages. It does not require a detailed model of the aircraft to be tuned, and its robustness in the face of UAV disturbances (e.g., variations in lift or drag) is better than the LQR. However with the PID sampling time being reduced, to save power, results from the averaged model show that for values higher than $2 \mathrm{~s}(0.5 \mathrm{~Hz})$ leads to large settling times, some minutes, and if too high, instability results. 


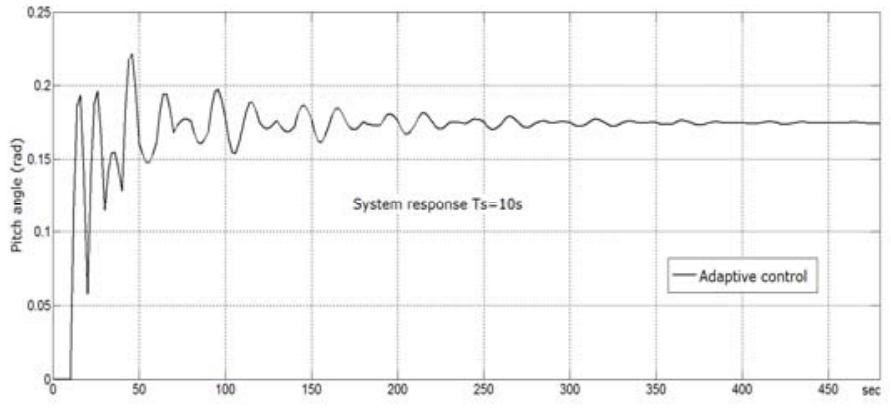

Fig. 10 Adaptive controller response for $\mathrm{Ts}=10 \mathrm{~s}$.

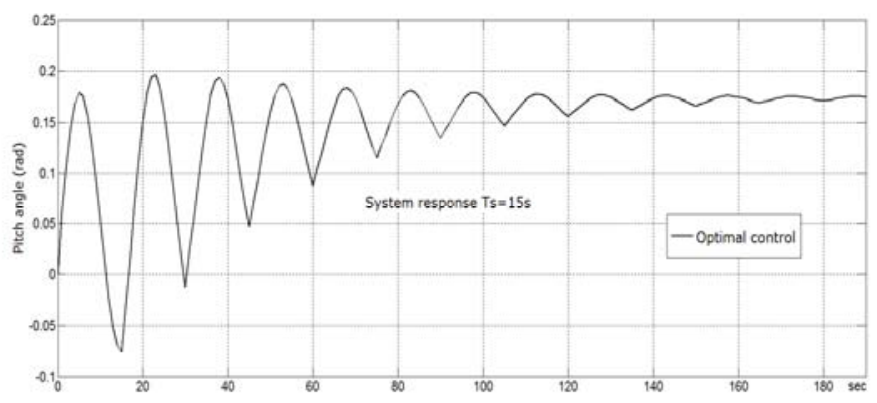

Fig. 11 Optimal (LQR) response for $\mathrm{Ts}=15 \mathrm{~s}$.

On the assumptions that the UAV model is sufficiently detailed and that the flight is not too perturbed (i.e., aircraft derivatives are constant), then the LQR is the best controller. In this scenarios where these conditions are satisfied, the LQR performance is best when using large sampling periods, and hence has the potential to yielding best percentage power consumption reduction. However as such scenarios are unrealistic in practice, relying on a direct use of LQR is risky and unadvisable. Further, LQR controllers are based in inner control loops, with a requirement for additional transducers, there is a consequent cost increment and safety reduction, e.g., a reliability dependency of a larger set of transducers.

Finally, the robustness of adaptive control technique (MRAS) allows enlarged sampling times, not much lower than what is possible using LQR. Also it has performed well in the presence of flight disturbances. In the trade-off balance between power consumption and flight safety it can be considered to yield the best performance. Notably, its power consumption can be reduced in a factor ranging from 5 to 10 over that of the popular PID. Compared to a typical PID based UAV autopilot, working at sampling periods larger than $20 \mathrm{~Hz}$, the savings amount to this 1.4 to 4.5 watts; that is up to almost $10 \%$ of the total power consumed in a light UAV (i.e., one lower than 1 $\mathrm{Kg})$.

In Table III some main results are compared, using as reference a 4 watts commercial autopilot working at $100 \mathrm{~Hz}$, with the PID algorithm. The selected sampling times in the table are the ones producing angle errors lower than $2 \%$ and settling times faster than $30 \mathrm{~s}$.

The LQR power consumption benefit is not reported here as, for it, pitch angle precision is sacrificed when Ts is enlarged; e.g., even with a value of $\mathrm{Ts}=0.0024 \mathrm{~s}$, the angle error is $5 \%$. Position errors are compared in Fig. 12.
TABLE III

SAMPLING TIMES AND POWER SAVINGS FOR EACH CONTROL STRATEGY

\begin{tabular}{|c|c|c|c|}
\hline $\begin{array}{c}\text { CONTROL } \\
\text { STRATEGY }\end{array}$ & $\begin{array}{c}\text { SAMPLING } \\
\text { TIME (Ts) }\end{array}$ & $\begin{array}{c}\text { RESULTING } \\
\text { REDUCTION } \\
\text { POWER } \\
\text { (FROM A } \\
\text { REFEFRENCE } \\
\text { OF 4 WATTS) }\end{array}$ \\
\hline $\begin{array}{c}\text { Optimal } \\
\text { control }\end{array}$ & $0.0024 \mathrm{~s}(416 \mathrm{~Hz})$ & No reduction & $\begin{array}{c}\text { Increased to } \\
16.6 \mathrm{~W}\end{array}$ \\
\hline PID control & $0.0142 \mathrm{~s}(70 \mathrm{~Hz})$ & $29.6 \%$ & $2.8 \mathrm{~W}$ \\
\hline $\begin{array}{c}\text { Adaptive } \\
\text { control }\end{array}$ & $1 \mathrm{~s}(1 \mathrm{~Hz})$ & $99 \%$ & $0.04 \mathrm{~W}$ \\
$0.65 \mathrm{~s}(1.5 \mathrm{~Hz})$ & $98.4 \%$ & $0.062 \mathrm{~W}$ \\
\hline
\end{tabular}
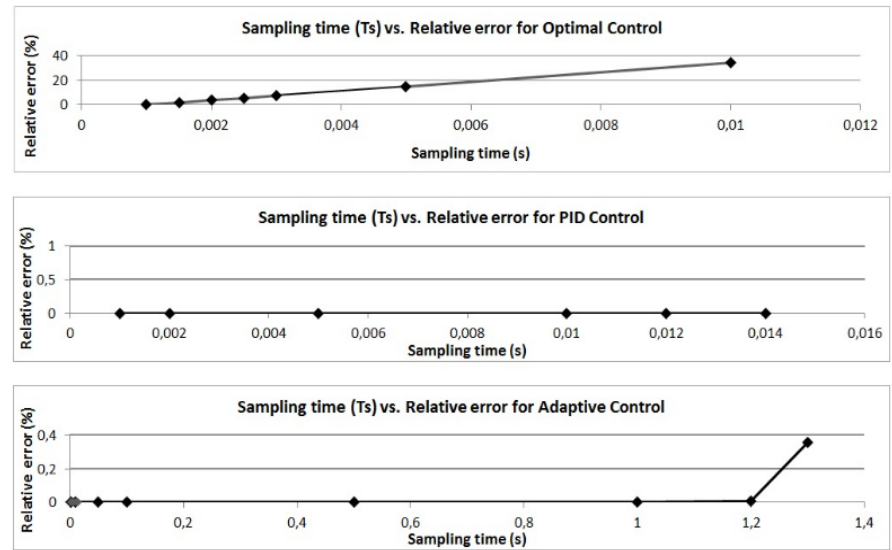

Fig. 12 Relative error vs. sampling time for the three control techniques

\section{CONCLUSION}

A study of digital control strategy designs for autopilots in small fixed wing UAVs with a particular focus on the consequences of particular designs on system stability and power consumption efficiency, simultaneously, has been presented.

To create a reasonable comparison framework, the computation of an 'averaged model' of reduced size and fixedwing UAVs was proposed and employed in order to study and comparatively evaluate stability and power consumption sensitivities to reductions in the sampling period. The proposition of such an 'averaged model' was deemed justified due to the variety of particular UAV linked to different control strategies and, in many cases, in the published literature, the absence of sufficient UAV design detail.

This 'average model' was obtained by merging compatible published models with others constructed from actual UAVs using the DATCOM software. An advantage of such a model is its independence of a particular UAV airframe. Hence the study results presented here have the advantage of some generality and thus usefulness as a first stage control algorithm evaluation in design studies when considering what to use for the autopilot in an actual UAV.

Among the studied strategies, PID and adaptive MRAS strategies were found to be the most independent of the UAV model details. In practice, nonetheless, this fact is complicated because the stability models are just an approximation and relying on the supposition of a t-invariant model is not too 
realistic in a context of weight variations, air pressures and altitudes, winds, stall curves, etc.

Considering both quality of flight and the enlargement of the sampling period, optimal LQR strategies are best. However they have poor robustness (lack of precision) when faced with disturbances. Besides, they need a complex space state model and internal loops, a fact which increases cost and reduces reliability.

Comparing PID and adaptive MRAS, the advantage of the latter is the possibility of significant reduction of power consumption, by between 5 and 10 times, as well greater robustness in their toleration of flight disturbances.

Our study considered the longitudinal dynamics of the aircraft. Following very similar lines, the assessment could be extended to the lateral dynamics, and our expectation is that this would yield similar qualitative conclusions about the control strategies.

\section{ACKNOWLEDGMENT}

The authors would like to thank Dr. Máirtín O’Droma (University of Limerick) for his help and constructive criticism in the review and edition of this paper.

\section{REFERENCES}

[1] Armstrom Flight Research Center Platforms [Onbline]. Available: http://www.nasa.gov/centers/armstrong/aircraft/index.html\#.VJL9vShC P8

[2] A. Aragon-Zavala, J.L Cuevas-Ruíz,J.A Delgado-Penín, High-Altitude Platforms for Wireless Communications, John Wiley \& Sons, 2008.

[3] Chunbo Luo ; McClean, S.I. ; Parr, G. ; Teacy, L., UAV Position Estimation and Collision Avoidance Using the Extended Kalman Filter, IEEE Trans. on Vehic. Technol., Volume: 62, Issue: 6, July 2013, pp $2749-2762$.

[4] Chao, H., Cao, Y. and Chen, Y., Autopilots for Small Unmanned Aerial Vehicles: A Survey, International Journal of Control, Automation and Systems, 8(1), 36-44, 2010

[5] Lizarraga, M., Curry, R. and Elkaim, G., Reprogrammable UAV Autopilot System (part 1), Feature Article, Circuit Cellar, Issue 249, April 2011

[6] E. Bertran; J.A. Delgado, Effects of packets format and SBAS measurement rate on the emergency control of an UAV", DASIA-02, Dublin, May, 2002.

[7] ICAO Manual on Remotely Piloted Aircraft Systems (RPAS, First edition, 2 April 2012

[8] J.Meyer, F. du Plessis, W. Clarke, "Design Considerations for Long Endurance Unmanned Aerial Vehicles ". InTech, Open Access, Available:http://www.intechopen.com/books/aerial_vehicles/design_co nsiderations for long endurance unmanned aerial vehicles.

[9] Lizarraga, M., "Design, Implementation and Flight Verification of a Versatile and Rapidly Reconfiguration UAV GNC Research Platform", $\mathrm{Ph}$. D. thesis, University of California Santa Cruz, December 2009

[10] Erdos, D. and Watkins, S.E., "UAV Autopilot Integration and Testing", IEEE, Region 5 IEEE Conference. April 2008.

[11] UAV Navigation, AP04 - The autopilot solution for unmanned aircraft, UAV Navigation, 2009.

[12] McDonnell Douglas Astronautics Company, The USAF Stability And Control Datcom -Volume I, User's Manual-, Missouri, 1979.

[13] Holy Cows, Inc., Datcom + Pro User's Manual-Version 3.0, Holy Cows, Orlando, 2011.

[14] Triputra, F.R., Trilaksono, B.R., Sasongko, R.A. and Dahsyat, M., "Longitudinal Dynamics System Modeling of a Fixed-Wing UAV towards Autonomous Flight Control System Development. -A Case Study of BPPT Wulung UAV Platform-“", International Conference on System Engineering and Technology, 2012.

[15] Sufendi, Trilaksono, B.R, Nasuition, S.H. and Purwanto, E.B. "Design and Implementation of Hardware-In-The-Loop-Simulation for UAV
Using PID Control Method", 3rd International Conference on Instrumentation, Communications, Information Technology and Biomedical Engineering (ICICI-BME), 2013.

[16] Huang, C., Shao, Q., Jin, P., Zhu, Z. and Zhang, B., "Pitch Attitude Controller Design and Simulation for a Small Unmanned Aerial Vehicle", International Conference on Intelligent Human-Machine Systems and Cybernetics, IEEE, 2009.

[17] Blakelock, J.H., Automatic Control of Aircraft and Missiles (Second Edition)", John Wiley \& Sons, Inc., United States, 1991.

[18] Nelson, R.C., Flight Stability and Automatic Control (Second Edition), The McGraw-Hill Companies, United States, 1998

[19] INTA. National Institute for Aerospace Technology (Spain) . Technical Information: High technology programs. Unmanned Aircraft. [Online]. Available:http://www.inta.es/programasaltatecnologia.aspx?Id=1\&SubI $\mathrm{d}=3$

[20] Protech data. [Online]. Available: http://www.galaxia.co.il/protech.htm

[21] J. Tristancho, M. Quilez, E.Pastor, "An Electromagnetic Interference Reduction Check List For Unmanned Aircraft System", 48th AIAA Aerospace Sciences Meeting Including the New Horizons Forum and Aerospace Exposition, 4 - 7 January 2010, Orlando, Florida

[22] ICARUS team, Shadow Flight Manual, 2009.

[23] Global Security Org. Global Hawk. [Online[. Available: http://www .globalsecurity.org/intell/systems/global hawk-pics.htm

[24] European Framework, HeliNet Project, European Framework Program 5, 2000-2003.

[25] Landau, Y. D., Adaptive Control, Marcel Dekker, New York. 1978.

[26] I.D. Landau, R. Lozano, M. M'Saad A. Karim. Adaptive Control: Algorithms, Analysis and Applications. Springer-Verlag. London. 2011.

[27] Texas Instruments, CMOS Power Consumption and Cpd Calculation, Texas Instruments Incorporated, 1997.

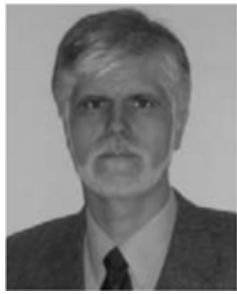

Eduard Bertran (M'91-SM'02) received the Engineer and Doctor Engineer degrees in telecommunication from the Technical University of Catalonia (UPC), Barcelona, Spain, in 1979 and 1985, respectively. He joined the Department of Signal Theory and Communications, where he is currently a Full Professor. His research interests include control, signal processing, radiocommunication electronics and avionics. He has served as associate director for Aeronautical Studies at the School of Telecommunications and Aerospace Engineering, as well as Secretary of the Spanish Section of the IEEE. He has been the coordinator of the Doctorate Program in Aerospace Science and Technology. He has collaborated in different national and European research projects and teams, and he has also collaborated in industrial projects. He is author or co-author of a number of journal and conference papers, as well as books and book chapters.

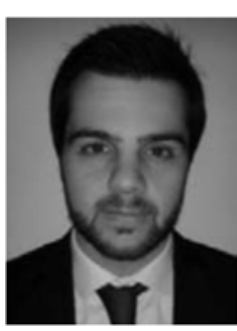

Alex Sànchez-Cerdà was born in Barcelona, in 1992. He received the M.S. degree in Aerospace Engineering from the Technical University of Catalonia (UPC) in 2014. During his studies he held a grant from the Government of Catalonia. He is currently working in a private company as a Test Engineer. His research interest are aerospace, UAVs, control strategies and 